\title{
Expanded temperature range of cholesteric blue phase by three dimensional network structures
}

\author{
Masayoshi Ojima, Takeshi Noma, Hiroaki Asagi, Akihiko Fujii, \\ Masanori Ozaki and Hirotsugu Kikuchi * \\ Grad. School of Engineering, Osaka Univ. 2-1 Yamada-oka, Suita, Osaka, Japan \\ e-mail: mojima@opal.eei.eng.osaka-u.ac.jp \\ * Institute for Materials Chemistry and Engineering, Kyushu Univ. 6-1 Kasuga-Koen, Kasuga, Fukuoka, Japan
}

Temperature range of cholesteric blue phase (BP) was expanded by infiltrating liquid crystal (LC) that exhibits blue phases into the three dimensional structures such as polymer network (PN) and mixed cellulose ester membrane (MCEM). Reflection spectrum and dielectric properties of the expanded BP have been measured. Cooling rate dependence of the BP temperature range was also investigated, which indicated that the expansion of the $\mathrm{BP}$ temperature range upon infiltrating LC into the three dimensional structures was induced by the pinning effect. The expansion of the BP temperature range was induced by pinning effect on the network surfaces of these three-dimensional structures. In particular, the temperature range of $\mathrm{BP}$ I in the LC/PN composite has cooling rate dependence and is six times wider than that of pure BP LC compound at $0.1{ }^{\circ} \mathrm{C} / \mathrm{min}$ cooling rate. Temperature range of BP in MCEM coated with other polymer was wider than that of in MCEM without polymer. The interaction between the surface of structure and LC molecules was affect the pinning power.

Key words: cholesteric, blue phase, three-dimensional structure, membrane, pinning effect

\section{INTRODUCTION}

The cholesteric blue phases (BPs) have three-dimensional (3D) helical structures and appear in temperature range between a chiral nematic phase and an isotropic liquid phase [1]. Theoretical and experimental works have demonstrated that chiral nematic liquid crystals with short pitch can form up to three distinct BPs, blue phase I (BPI), blue phase II (BPII) and blue phase III (BPIII). Their 3D structures have the lattice constant in the order of visible wavelength and include regularly ordered disclinations resulting from the $3 \mathrm{D}$ helicoidal molecular alignment $[2$, $3]$. From the viewpoint of display application, since BPs are optically isotropic, display devices based on them should have a wide view angle and should not require an alignment process. In addition, because of their $3 \mathrm{D}$ helical structure with a periodicity in the order of visible wavelength, applications to photonic crystals [4-6] and fast electro-optical modulation [7-9] are also expected.

Despite their potential applications, BPs appear in very narrow temperature range because of the inevitable existence of disclination. Recently, several attempts have been proposed to widen the temperature range of the BPs, which describe the stabilizations of the 3D cubic lattice by polymerizing disclination lines [10], and by using a flexoelectricity of twin molecules [11] and a chiral compound possessing molecular biaxiality [12]. We have proposed also the stabilization by confining $\mathrm{BPs}$ in $3 \mathrm{D}$ polymer network (PN) structure that is formed by a polymerization-induced phase separation of the liquid crystal - prepolymer mixture [13]. These synthesize atmosphere leads to obscure mechanism of stabilization of BPs.
In this study, the temperature range of the BP in the PN fabricated with phase separation and infiltrated in MCEM was discussed. Infiltrating the BP into the almost same structure as the fabricated structure with phase separation leads also expansion of the temperature range of BP I, and the expansion became wider with coating the polymer on the surface of MCEM.

\section{EXPERIENCE}

The liquid crystal (LC) materials used in this study comprised a nematic mixture (JC-1041XX, Chisso Co.) and 4-cyano-4'-pentylbiphenyl (5CB). A chiral dopant (ISO-(6OBA)2) was used to induce the BPs and a cholesteric phase. The constituent fractions and the phase-transition temperatures of the LC are listed in Tables I and II.

Table I Constituent fraction of the BP

\begin{tabular}{|c|c|c|}
\hline LC mixture & 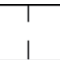 & Chiral dopant \\
\hline JC1041XX & I & ISO- $(6 \mathrm{OBA})_{2}$ \\
\hline$\overline{46.5 \mathrm{wt} \%}--\overline{46.5 \mathrm{wt} \%}-$ & & $-\overline{7.0 \mathrm{wt} \%}--$ \\
\hline
\end{tabular}

Table II Phase-transition temperatures of the BP

\begin{tabular}{|c|c|c|}
\hline \multicolumn{3}{|c|}{ Phase transition temperature } \\
\hline \multicolumn{3}{|c|}{$\mathrm{Ch}-42.3^{\circ} \mathrm{C}-\mathrm{BP}$ I- $44.0^{\circ} \mathrm{C}-\mathrm{BP}$ II- $45.2^{\circ} \mathrm{C}$-ISO } \\
\hline \multirow{2}{*}{ Temperature range } & BP I & $1.7^{\circ} \mathrm{C}$ \\
\hline & BP II & $1.2^{\circ} \mathrm{C}$ \\
\hline
\end{tabular}

To form a PN in the LC, NOA65 (Norland Products) 
was used as a UV-curable monomer. Homogeneous mixtures of $\mathrm{LC}$ and monomer were prepared at ratio 2 : 1. A glass cell was filled with a homogeneous mixture and irradiated with UV light (365 nm) passing through a bandpath UV filter for $10 \mathrm{~min}$ at room temperature to induce a phase separation between the LC and the polymer.

Figure 1 shows the scanning electron microscope (SEM) images of fabricated polymer network structure and MCEM having 1- $\mu \mathrm{m}$ pore size. The morphology of the polymer network depends on the constitution fraction of the LC and monomer. The averaged size of the vacant spaces in the PN is approximately $5 \mu \mathrm{m}$, therefore these three-dimensional structure was almost same random network structure.

The LC materials were infiltrated into the MCEM in vacuumed chamber.
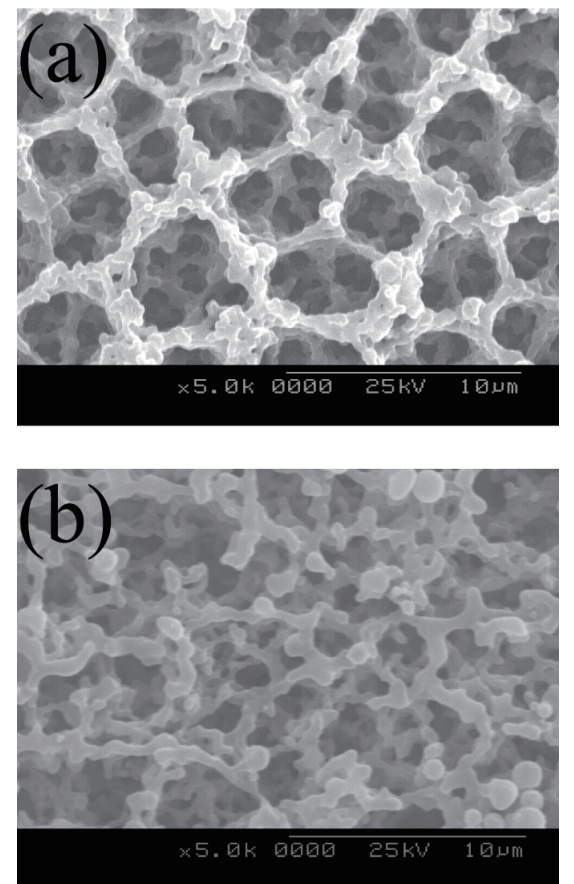

Figure 1 SEM images of (a) fabricated polymer network and (b) MCEM having 1- $\mu \mathrm{m}$ pore size

UV curable monomer used for coating MCEM comprised 2-ethylhexyl acrylate (EHA, Aldrich) and diacrylate monomer (RM257, Merck). 2,2-Dimethoxy-2-phenyl acetophenone (DMPAP, Aldrich) was added to the solution as a photo-initiator. The constituent fractions of monomer and photo-initiator were listed in Table III. A solution of the monomer in toluene $(10 \mathrm{wt} \%)$ infiltrated into the MCEM and then toluene was evaporated in vacuumed chamber. After evaporation, the sample was irradiated with UV light $(365 \mathrm{~nm})$ at $90^{\circ} \mathrm{C}$ in air.

Table III Constituent fractions of the UV curable monomer and photo-initiator

\begin{tabular}{|c|c|c|}
\hline \multicolumn{2}{|c|}{ UV curable monomer } & Photo-initiator \\
\hline EHA & RM257 & DMPAP \\
\hline$\overline{90 \overline{\mathrm{mol}} \%}$ & $\overline{10 \mathrm{~mol} \%}$ & $5 \mathrm{~mol} \%$ to monomer \\
\hline
\end{tabular}

The optical textures of the samples were observed by a polarizing optical microscope (Nikon) equipped with a hot stage (METTLER, FP90). The reflection spectrum of the samples was measured with a multichannel photodetector (Hamamatsu photonics C7473-36).

\section{RESULTS AND DISCUSSION}

3.1 Polarizing optical microscope observations

Polarizing optical microscope observations can show characteristic textures apparently associated with different kinds of liquid crystal phases. Figure 2 shows the optical texture of the LCs with PN and infiltrated in $1-\mu \mathrm{m}$ MCEM. These blue and green platelet textures are the specific characteristics of BP I. For the BP LC without the three-dimensional structures, on slowly cooling $\mathrm{LC}\left(0.01^{\circ} \mathrm{C} / \mathrm{min}\right)$ from the isotropic phase, the size of platelet textures becomes larger than that on faster cooling $\left(1.0^{\circ} \mathrm{C} / \mathrm{min}\right)$. However, the size of platelet of BP in the three-dimensional structures did not depend on cooling rate. This result indicates that platelet was confined in the voids of these structures.
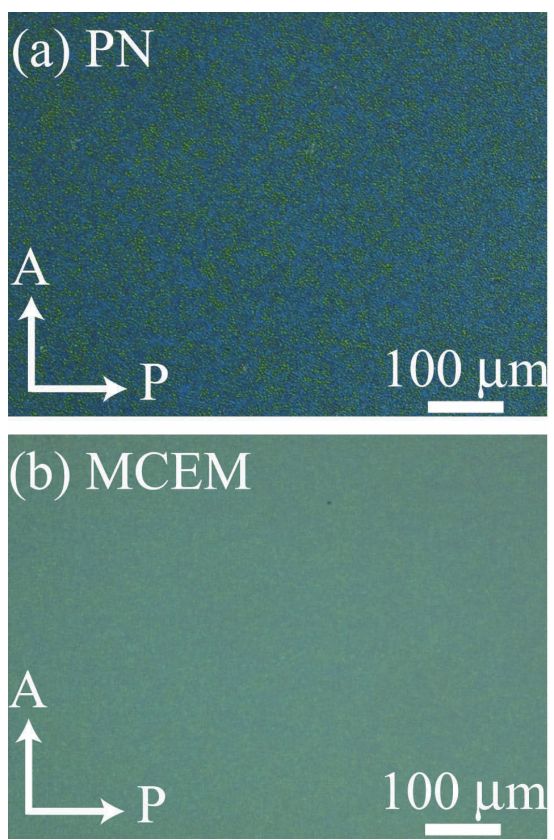

Figure 2 Optical textures of the LC

(a) with PN and (b) infiltrated in 1- $\mu \mathrm{m}$ MCEM

\subsection{Reflection spectrum}

It is well known that there are three thermodynamically stable blue phases, BP I, BP II and BP III, occurring in that order with increasing temperature. BP I and BP II exhibit a three-dimensional periodic structure in the director field, having body-centered and simple cubic symmetries, respectively. The lattice periods are comparable to the wavelength of visible light, resulting in the Bragg reflection of visible light. Figure 3 shows the reflection spectrum of the LC infiltrated in 1- $\mu \mathrm{m}$ MCEM at various temperatures. Reflection peak was observed at $520 \mathrm{~nm}\left(41.0^{\circ} \mathrm{C}\right)$ and $425 \mathrm{~nm}\left(47.0^{\circ} \mathrm{C}\right)$. These reflection peaks could be assigned to be the Bragg diffraction from the (110) plane of the cubic lattice in BP I and from the (100) plane of the cubic lattice in BP II, respectively. 
Therefore, it is concluded that LC exhibits BP even in the MCEM. These Bragg reflection peaks were also observed from the BP in the polymer network.

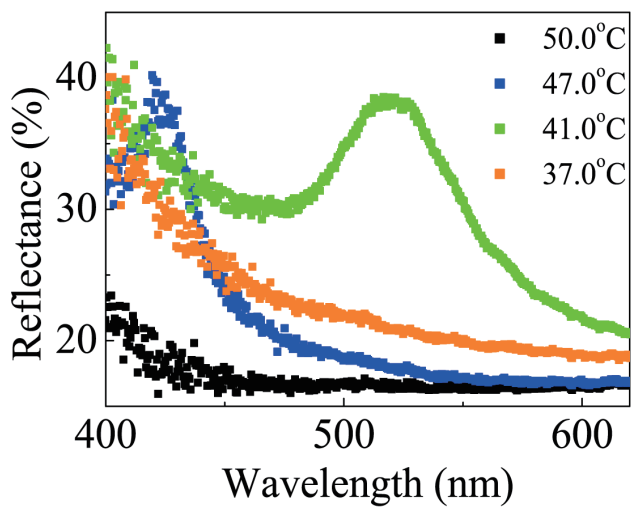

Figure 3 Reflective spectra of the BP infiltrated in 1- $\mu \mathrm{m}$ MCEM at $50.0^{\circ} \mathrm{C}$ (Isotropic), $47.0^{\circ} \mathrm{C}$ (BP II), $41.0^{\circ} \mathrm{C}$ (BP I) and $37.0^{\circ} \mathrm{C}$ (cholesteric)

From the polarizing optical microscope observations and reflection spectrum measurement, cooling rate dependence of the temperature range of the BP I of LC with PN and infiltrated in $1-\mu \mathrm{m}$ MCEM was summarized in Figure 4. The BP I temperature range depends on the cooling rate, while that of BP II stayed constant. When the cooling rate is enormously slow, the $\mathrm{BP}$ I range of the LC with PN will be approximately $4^{\circ} \mathrm{C}$, while that of the BP LC without structure will be approximately $1^{\circ} \mathrm{C}$. These results also support that the intrinsic temperature range of $\mathrm{BP}$ is expanded by the existence of the PN. Temperature range of BP infiltrated in MCEM is approximately the same as that of BP LC without MCEM when the cooling rate is enormously low. However, temperature range of BP infiltrated in MCEM become gradually wider with faster cooling rate. These results indicate that the expanded BP in the MCEM is not thermodynamically stable and temperature range of $\mathrm{BP}$ in MCEM is expanded by pinning effect of membrane structure. These results also indicate that the temperature range of $\mathrm{BP}$ is expanded with not only phase separation method but also infiltration method.

It should be noted that the temperature range of the BP I is expanded during cooling process. However this was not observed during heating process. These results indicate that tree-dimensional structure causes a pining effect on appearance of BP I. The temperature range of BP I expanded by tree-dimensional structure was much wider than the expanded temperature range by the super cooling of BP LC without MCEM [14].

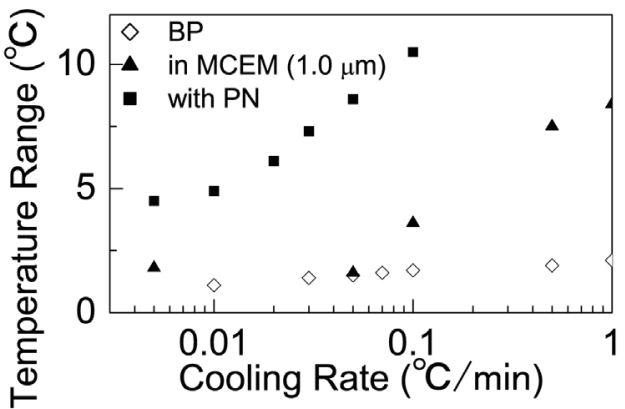

Figure 4 Cooling rate dependence of the temperature range of BP I with PN, infiltrated in 1- $\mu \mathrm{m}$ MCEM and

BP LC without the three-dimensional structures

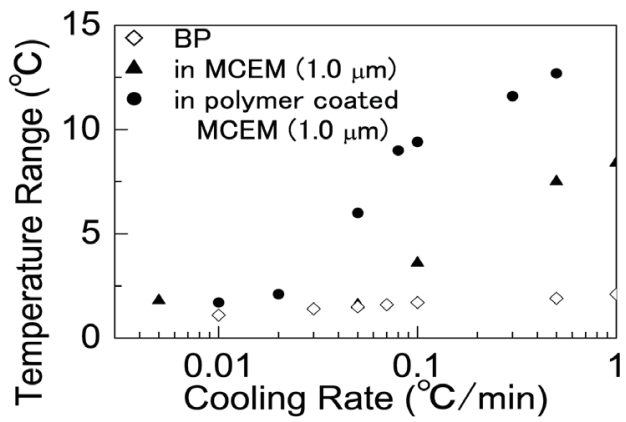

Figure 5 Cooling rate dependence of the temperature range of BP I infiltrated in 1- $\mu \mathrm{m}$ MCEM, infiltrated in polymer coated MCEM and BP LC without the three-dimensional structures

\subsection{Polymer coat effect}

Polymer coat effect to the BP expansion has been investigated. UV curable monomer was coated and then polymerized on the surface of network structure of MCEM. According to the SEM observation, the pore structure of polymer coated MCEM was same as non-coated MCEM. It is considered that expanded temperature range will be changed depend on the interaction between the surface of the structure and LC molecules. Figure 5 shows cooling rate dependence of the temperature range of $\mathrm{BP} I$ infiltrated in polymer-coated MCEM. The temperature range of BP I infiltrated in polymer-coated MCEM became wider than that of uncoated MCEM at faster cooling rate region. When the cooling rate is enormously slow, the temperature rage was also approximately same as that of BP LC without MCEM. These results indicate that the polymer coating enhances the pinning power, but does not affect the intrinsic temperature range of the BP. Polymer coat effect with other polymers is under consideration.

\section{CONCLUSION}

The temperature range of the BP I was expanded at the cooling stage by infiltrating the BPs in the three-dimensional structures, such as PN and MCEM. On the heating stage, there was no expansion in the temperature range of the BPs. The expansion temperature range depended on cooling rate. Therefore we considered that this effect in expansion of the temperature range was induced by the pinning effect on 
the surface of the structures. The pinning effect became larger with the polymer coating on the three-dimensional structure.

ACKNOWLEDGMENT

We acknowledge Chisso Co. Ltd. for kindly providing the nematic liquid crystal material. This work was supported by a Grant-in-Aid for Japan Society for the Promotion of Science (JSPS) Fellows from The Ministry of Education, Culture, Sports, Science and Technology, Japan (No. 195610) and Photonics Advanced Research Center of Osaka University.

\section{REFERNCES}

[1] D. C. Wright and N. D. Mermin, Rev. Mod. Phys., 61, 385 (1989).

[2] D. L. Johnson, J. H. Flack, and P. P. Crooker, Phys. Rev. Lett., 45, 641 (1980).

[3] S. Meiboom, M. Sammon, and W. F. Brinkman, Phys. Rev. A, 27, 438 (1983).

[4] P. Etchegoin, Phys. Rev., E 62, 1435 (2000).

[5] W. Cao, A. Munoz, P. Palffy-Muhoray and B. Taheri, Nat. Mater., 1, 111 (2002).

[6] S. Yokoyama, S. Mashiko, H. Kikuchi, K. Uchida and T. Nagamura, Adv. Mater., 18, 48 (2006).

[7] Y. Hisakado, H. Kikuchi, T. Nagamura and T. Kajiyama, Adv. Mater., 17, 2311 (2005).

[8] Y. Haseba, H. Kikuchi, T. Nagamura and T. Kajiyama, Adv. Mater., 17, 2311 (2005).

[9] Y. Haseba and H. Kikuchi, Mol. Cryst. Liq. Cryst., 470, 1, (2007)

[10] H. Kikuchi, M. Yokota, Y. Hisakado, H. Yang, and T. Kajiyama, Nat. Mater., 1, 64 (2002).

[11] H. J. Coles and M. N. Pivnenko, Nature, 436, 997 (2005).

[12] A. Yoshizawa, M. Sato, and J. Rokunohe, J. Mater. Chem., 15, 3285 (2005).

[13] T. Noma, M. Ojima, H. Asagi, Y. Kawahira, A. Fujii, M. Ozaki and H. Kikuchi, e-J. Surf. Sci. Nanotech., 6, 17 (2008).

[14] E. Demikhov, H. Stegemeyer, and V. Tsukruk, Phys. Rev. A, 46, 4879-4887 (1992).

(Received December 9, 2008; Accepted March 15, 2009) 